\title{
Impact of Traumatic Dental Injury on the Quality of Life of Brazilian Preschool Children
}

Maria B. Siqueira ${ }^{1, \dagger}$, Ramon T. Firmino ${ }^{1, \dagger}$, Marayza A. Clementino ${ }^{1, \dagger}$, Carolina C. Martins ${ }^{2, \dagger}$, Ana F. Granville-Garcia ${ }^{1, \dagger}, *$ and Saul M. Paiva ${ }^{2, \dagger}$

1 Department of Dentistry, School of Dentistry, State University of Paraíba, Av. das Baraúnas, 351, Campina Grande, Paraiba 58109753, Brazil; E-Mails: mbldsiqueira@yahoo.com.br (M.B.S.); ramontargino@gmail.com (R.T.F.); marayza84@gmail.com (M.A.C.)

2 Department of Pediatric Dentistry and Orthodontic, School of Dentistry, Federal University of Minas Gerais, Av. Antônio Carlos, 6627 Belo Horizonte, Minas Gerais 31270901, Brazil; E-Mails: carolcm10@hotmail.com (C.C.M.); smpaiva@uol.com.br (S.M.P.)

$\dagger$ These authors contributed equally to this work.

* Author to whom correspondence should be addressed; E-Mail: anaflaviagg@gmail.com; Tel.: +55-83-3315-3326.

Received: 25 July 2013; in revised form: 18 October 2013 / Accepted: 8 November 2013 / Published: 28 November 2013

\begin{abstract}
This study aimed to assess the impact of traumatic dental injury (TDI) on the quality of life of preschoolers and their families. A cross-sectional study was carried out, with a sample of 814 children, aged three to five years old, in Campina Grande, Brazil. Parents/caregivers were asked to complete the Brazilian Early Childhood Oral Health Impact Scale and a questionnaire on socio-demographic data. Oral examinations of the children were performed by three previously calibrated dentists. Bivariate and multiple Poisson regression analyses were performed $(\alpha=5 \%)$. The prevalence of negative impact from oral conditions on quality of life was $31.1 \%$ among the children and $24.7 \%$ among the families. TDI was not associated with a negative impact on quality of life. Parent/caregiver's assessment of the child's oral health (PR $=1.210 ; 95 \%$ CI: $1.027-1.426)$ and history of toothache ( $\mathrm{PR}=4.997 ; 95 \% \mathrm{CI}$ : 2.943-8.493) remained in the final model for the child section, whereas only a history of toothache $(\mathrm{PR}=2.791 ; 95 \% \mathrm{CI}$ : 1.801-4.325) remained in the final model for the family section. TDI exerted no negative impact on quality of life in the present sample. A history of toothache was the only
\end{abstract}


variable associated with a negative impact on the quality of life of the preschoolers and their families.

Keywords: quality of life; tooth injuries; child

\section{Introduction}

Current studies stress the need to consider the functional and psychosocial dimensions of oral health for the implementation and evaluation of public health interventions in dentistry [1]. The assessment of quality of life has become an integral part of the evaluation of health programs, as traditional dental indicators focused on the presence/absence of oral disease do not demonstrate the extent to which such conditions exert an effect on activities of daily living [2,3]. Thus, in addition to clinical measures, information on oral health-related quality of life (OHRQoL) is essential for healthcare policy makers to perform an adequate assessment of oral health needs [4].

Oral diseases and disorders can have an impact on the quality of life of preschool children and their parents, affecting their oral health and wellbeing [5]. The Early Childhood Oral Health Impact Scale (ECOHIS) is a proxy measure for assessing the impact of oral health problems on the quality of life of preschool children and their families in epidemiological surveys [6-8]. Parents play an important role in decision-making with regard to their children's oral health and this assessment tool measures parents' perceptions on how oral health problems, including symptoms, the disease itself, and its treatment, affect their child's quality of life [6,9].

With the decline of the prevalence of dental caries, public oral health for children has become more concerned with other oral health issues such as dental trauma injury (TDI) [10], which is the second most prevalent type of dental condition affecting children aged five years or younger [11-15]. TDI can result in pain, loss of function, emotional distress, and can adversely affect the developing occlusion as well as dental esthetics, with a negative impact on the lives of children [16,17]. Few studies have been carried out in Brazil assessing the impact of TDI on the quality of life of preschool children and their families [17,18]. Moreover, there is no consensus with regard to the findings and no population-based studies have been conducted. Thus, little is known regarding the feelings of children with TDI or the emotional and psychological impact of this condition on young children and their families.

The purpose of the present study was to assess the impact of traumatic dental injury on the quality of life of preschool children and their families in northeastern Brazil.

\section{Methods}

\subsection{Sample Characteristics}

A population-based, cross-sectional study was carried out involving 814 male and female children aged three to five years enrolled at preschools (both public and private) in the city of Campina Grande, Brazil. Participants were selected from a total population of 12,705 children in this age group and corresponded to $6.41 \%$ of that population. Campina Grande (population: 386,000 ) is an industrialized city in northeastern Brazil divided into six health districts. The city has considerable cultural, social, 
and economic disparities, with a mean monthly income of approximately US\$110 per capita and a Human Development Index of 0.72 [19].

A two-phase random sampling strategy was used to ensure representativeness. In the first phase, preschools were randomly selected from each health district, and, in the second phase, children were randomly selected from each preschool. Eighteen of the 127 public preschools and fifteen of the 122 private preschools in the city of Campina Grande were randomly selected. The sample size was calculated based on a four percent margin of error, a ninety-five percent confidence level and a fifty percent prevalence rate of impact on child and family OHRQoL. A correction factor of 1.2 was applied to compensate for the design effect [20]. The minimum sample size was estimated at 720 schoolchildren, to which a further twenty percent was added to compensate for possible losses, giving a total sample of 864 schoolchildren, who were randomly selected from the previously selected schools for participation in the study.

\subsection{Eligibility Criteria}

The following were the inclusion criteria: age three to five years old; enrollment in a preschool or daycare center; absence of systemic disease according parent/caregiver's information; being accompanied by a Brazilian Portuguese language-speaking caregiver; agreement to participate through a signed statement of informed consent; and the return of completed questionnaires. The exclusion criterion was having four missing maxillary incisors due to caries or physiological exfoliation, which could compromise the clinical diagnosis of TDI.

\subsection{Training and Calibration Exercise}

The calibration exercise consisted of two steps, both theoretical and clinical. The theoretical step involved a discussion of the criteria for the diagnosis of TDI and malocclusion, the administration of the ICDAS II, and an analysis of photographs. A specialist in pediatric dentistry (the gold standard in this theoretical framework) coordinated this step, instructing three general dentists on how to perform the examination. Cases of disagreement were discussed with the group of dentists who participated in the exams prior to the clinical step. The clinical step was performed at a randomly selected preschool that was not part of the main sample. Each dentist examined 50 previously selected children between three to five years of age. Inter-examiner agreement was tested by comparing each examiner with the gold standard ( $\mathrm{K}=0.85$ to 0.90$)$. A seven-day interval was respected between clinical examinations for the determination of intra-examiner agreement $(K=0.85$ to 0.90$)$. Data analysis involved Cohen's Kappa coefficient occurred on a tooth-by-tooth basis. As Kappa coefficients were very good [21], the examiners were considered capable of performing the epidemiological study.

\subsection{Pilot Study}

A pilot study was performed to test the methodology and comprehension of the questionnaires. The children in the pilot study $(n=40)$ were not included in the main sample. As there were no misunderstandings regarding the questionnaires or the methodology, no changes needed to be made to the data collection process. 


\subsection{Non-Clinical Data Collection}

The Early Childhood Oral Health Impact Scale (ECOHIS) and questionnaires addressing socio-demographic and health data of children questionnaires were filled out by parents/caregivers. The ECOHIS assesses parents/caregivers' perceptions regarding the negative impact of oral health problems on the quality of life of preschool children and their families. This scale is divided into two sections; child impact and family impact, with six domains and thirteen items. The domains in the Child Impact Section are symptoms (one item), function (four items), psychological (two items) and self-image/social interaction (two items). The domains in the Family Impact Section are distress (two items) and family function (two items). Each item has six response options: $0=$ never, $1=$ hardly ever, 2 = occasionally, $3=$ often, $4=$ very often and $5=$ don't know. Item scores are summed for each section (“don't know" responses are not counted). The total score ranges from 0 to 36 in the Child Impact Section and 0 to 16 in the Family Impact Section, with higher scores indicating greater impacts and/or more problems. The Brazilian version of the ECOHIS has been validated in Brazilian Portuguese and used in previous studies $[8,18,22]$. There were two dependent variables: the impact on children's OHRQL and impact on the family's OHRQL. The presence of impact on children's QoL was considered when at least one answer "hardly ever" was giver in any of four domains (symptom, functional, psychological, self-image/social). The absence of impact was when all answers were given as "never". The presence of impact of a family's QoL was considered when at least one answer of "hardly ever" was given in any of two domains (distress and family function). An absence of impact on family' QoL was when all domains were answered "never" [6,17,23,24].

Socio-demographic data: Parent/caregiver's age and years of schooling, number of people in the home; type of school; monthly household income (categorized based on the minimum salary in Brazil = US\$312.50)

Child health data: Parent/caregiver's assessment of child's general and oral health; history of toothache; history of dental visits; and history of trauma (parents/caregivers of children with a normative diagnosis of trauma).

\subsection{Clinical Data Collection}

The clinical examination was performed at the preschool after the return of the questionnaires and after having received signed informed consent. The examinations were performed by three dentists who had undergone the calibration exercise. Prior to the clinical exam, the children brushed their teeth under the examiner's supervision. For such, each child received a kit containing a toothbrush, toothpaste, and dental floss to remove bacterial biofilm from the dental surfaces and facilitate the diagnosis. Lip seal was evaluated prior to the intraoral examination without the subject aware that he or she was being observed and was determined adequate when contact between the lips occurred in the resting position with the teeth in occlusion [25].

Oral examinations were performed in the knee-to-knee position with the aid of a portable lamp attached to the examiner's head (Petzl Zoom head lamp, Petzl America, Clearfield, UT, USA). The dentists used individual cross-infection protection equipment as well as packaged, sterilized mouth mirrors (PRISMA ${ }^{\circledR}$, Sao Paulo, SP, Brazil), Williams' probes (WHO-621, Trinity ${ }^{\circledR}$, Campo Mourão, 
PA, Brazil) and dental gauze. To measure overjet, the examiner placed a Williams' periodontal probe on the incisal surface of the maxillary central incisors parallel to the occlusal plane to determine the horizontal relation of the incisors. This measurement was taken with the teeth in centric occlusion. Overjet was dichotomized as (i) $2 \mathrm{~mm}$ or less (normal overjet); and (ii) greater than $2 \mathrm{~mm}$ (accentuated overjet) [26]. Open bite was recorded when the anterior teeth were not in contact with the posterior teeth in occlusion [27]. The classification proposed by Andreasen et al. [28] was used for the clinical diagnosis of TDI: enamel fracture, enamel and dentin fracture, complicated crown fracture, extrusive luxation, lateral luxation, intrusive luxation, and avulsion. A visual assessment of tooth discoloration was also performed. Dental caries was diagnosed using the International Caries Detection and Assessment System (ICDAS II) [29]; the first visual change in enamel was considered as caries. Children with at least one of the following conditions were classified as having malocclusion: overbite [26], accentuated overjet [26,30], and posterior crossbite recognized first by Foster and Hamilton [31]. Following the examination, fluoride varnish was applied for all children and those with carious lesions or other dental needs were sent for treatment.

\subsection{Statistical Analysis}

Simple descriptive statistics were performed to characterize the sample and demonstrate the distribution of ECOHIS items. Bivariate analysis was performed using the chi-square test to determine associations between TDI and negative impacts on the ECOHIS items. Bivariate Poisson regression analysis with robust variance was employed to determine associations between the independent variables and negative impact on the quality of life on the children and their families $(p<0.05)$. Multivariate Poisson regression models were constructed after controlling for the confounding effects of dental caries and malocclusion. Forward stepwise multivariate Poisson regression models were constructed with variables having achieved a $p$-value $<0.20$ in the bivariate analysis as well as variables considered epidemiological determinants, after controlling for the confounding effects of dental caries and malocclusion. This analysis was performed with two dependent variables at a time; Impact on Quality of Life of the Child and Impact on Quality of Life of the Family. Data organization and statistical analyses were carried out using the Statistical Package for Social Sciences (SPSS for Windows, version 18.0, SPSS Inc., Chicago, IL, USA).

\subsection{Ethical Considerations}

The present study received approval from the Human Research Ethics Committee of the State University of Paraíba (Campina Grande, Brazil) under process number 00460133000-11 in compliance with Resolution 196/96 of the Brazilian National Health Council.

The flow chart of the study (Figure 1): 
Figure 1. Flowchart of study.

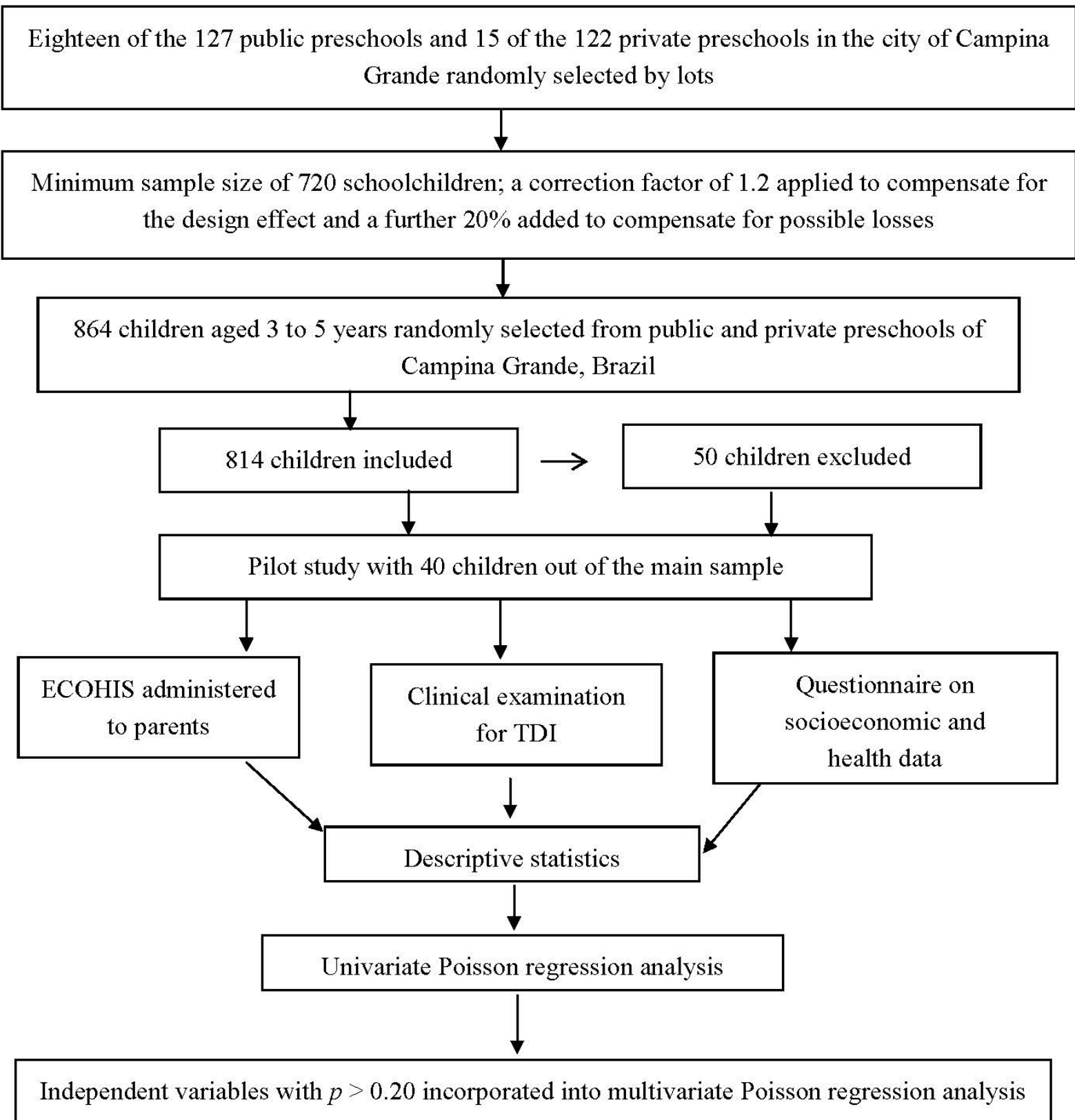

\section{Results}

Among the 864 selected, 814 participated in the present study, corresponding to $94.22 \%$ of the total determined by the sample calculation process. The exclusion of 50 children was due to a lack of participation on the part of the child for medical reasons (2), incomplete questionnaires (15), absence from preschool/daycare center on the days scheduled for the clinical exams (15), and a lack of cooperation during the clinical exam (18). Table 1 displays the socio-demographic and clinical data of the sample. The prevalence of TDI was $34.6 \%$. The upper central incisors were the most affected $(88.4 \%)$, followed by lateral central incisors $(8.9 \%)$. Only 10 affected teeth $(2.7 \%)$ were lower incisors. Enamel fracture was the most common type of TDI (17.0\%), followed by tooth discoloration (11.2\%). Most children had only one tooth affected (21.7\%). 
Table 1. Frequency distribution of preschool children according to independent variables.

\begin{tabular}{|c|c|c|}
\hline \multirow{2}{*}{ Variable } & \multicolumn{2}{|c|}{ Frequency } \\
\hline & $\mathbf{N}$ & $\%$ \\
\hline \multicolumn{3}{|l|}{ - Gender of child } \\
\hline Female & 392 & 48.2 \\
\hline Male & 422 & 51.8 \\
\hline \multicolumn{3}{|l|}{ - Number of residents in home } \\
\hline$<$ to 6 residents & 674 & 84.6 \\
\hline 6 or more residents & 123 & 15.4 \\
\hline \multicolumn{3}{|l|}{ - Household income } \\
\hline$>3$ times the minimum wage & 144 & 18.5 \\
\hline$\leq 3$ times the minimum wage & 634 & 81.5 \\
\hline \multicolumn{3}{|l|}{ - Parent/caregiver's schooling } \\
\hline$>8$ years of study & 437 & 54.0 \\
\hline$\leq 8$ years of study & 373 & 46.0 \\
\hline \multicolumn{3}{|c|}{ - Parent/caregiver's assessment of child's oral health } \\
\hline Good & 759 & 93.4 \\
\hline Poor & 54 & 6.6 \\
\hline \multicolumn{3}{|c|}{ - Parent/caregiver's assessment of child's general health } \\
\hline Good & 801 & 99.0 \\
\hline Poor & 8 & 1.0 \\
\hline \multicolumn{3}{|l|}{ - TDI } \\
\hline Yes & 281 & 34.6 \\
\hline No & 533 & 65.4 \\
\hline \multicolumn{3}{|l|}{ - Type of TDI } \\
\hline None & 533 & 65.4 \\
\hline Tooth discoloration & 91 & 11.2 \\
\hline Enamel fracture & 138 & 17.0 \\
\hline Enamel + dentin fracture & 39 & 4.8 \\
\hline Luxation & 9 & 1.1 \\
\hline Avulsion & 4 & 0.5 \\
\hline \multicolumn{3}{|c|}{ - Number of teeth affected by TDI } \\
\hline 2 or more teeth & 104 & 12.8 \\
\hline 1 tooth & 177 & 21.7 \\
\hline None & 533 & 65.5 \\
\hline \multicolumn{3}{|l|}{ - History of toothache } \\
\hline Yes & 71 & 36.6 \\
\hline No & 123 & 63.4 \\
\hline \multicolumn{3}{|l|}{ - History of dental visits } \\
\hline Yes & 196 & 24.2 \\
\hline No & 614 & 75.8 \\
\hline \multicolumn{3}{|l|}{ - Type of preschool } \\
\hline Public & 438 & 53.8 \\
\hline Private & 376 & 46.2 \\
\hline
\end{tabular}


The prevalence of negative impact on quality of life was $31.1 \%$ and $24.7 \%$ among children and their families, respectively. The items with the greatest frequency of the Child Impact Section of the ECOHIS were "related to pain" (21.6\%), "had difficulty eating some foods" (12.5\%), and "had difficulty drinking hot or cold beverages" $(12.4 \%)$. The items with the greatest frequency of the Family Impact Section were "felt guilty" (17.3\%) and "been upset" (14\%) (Table 2).

Table 2. Prevalence of impact of oral health on quality of life and ECOHIS scores among preschool children.

\begin{tabular}{lcccc}
\hline \multicolumn{1}{c}{ Domains, Items } & ECOHIS & & & \\
\hline & $\begin{array}{c}\text { SCORE } \\
\text { Mean } \pm \text { DP }\end{array}$ & $\begin{array}{c}\text { Minimum- } \\
\text { Maximum }\end{array}$ & $\begin{array}{c}\text { n (\%) } \\
\text { Don't Know }\end{array}$ & $\begin{array}{c}\text { n (\%) } \\
\text { Prevalence of } \\
\text { impact }\end{array}$ \\
\hline - Child Impact & $2.21 \pm 4.18$ & $0-31$ & - & $31.1 \%$ \\
\hline Related to pain & $0.58 \pm 0.979$ & $0-4$ & $14(1.7 \%)$ & $176(21.6 \%)$ \\
Had difficulty drinking hot & $0.35 \pm 0.816$ & $0-4$ & $10(1.2 \%)$ & $101(12.4 \%)$ \\
or cold beverages & $0.35 \pm 0.830$ & $0-4$ & $11(1.4 \%)$ & $103(12.5 \%)$ \\
Had difficulty eating some foods & $0.21 \pm 0.707$ & $0-4$ & $25(3.1 \%)$ & $59(7.2 \%)$ \\
Had difficulty pronouncing words & $0.12 \pm 0.466$ & $0-3$ & - & $29(3.6 \%)$ \\
Missed preschool, daycare or school & $0.17 \pm 0.596$ & $0-4$ & $8(1.0 \%)$ & $45(5.5 \%)$ \\
Had trouble sleeping & $0.29 \pm 0.745$ & $0-4$ & $8(1.0 \%)$ & $85(10.4 \%)$ \\
Been irritable or frustrated & $0.08 \pm 0.395$ & $0-4$ & $10(1.2 \%)$ & $18(2.2 \%)$ \\
Avoided smiling or laughing & $0.08 \pm 0.00$ & $0-4$ & $9(1.1 \%)$ & $19(2.3 \%)$ \\
Avoided talking & $1.13 \pm 2.167$ & $0-14$ & & $24.7 \%$ \\
\hline - Family Impact & $0.39 \pm 0.902$ & $0-4$ & $11(1.4 \%)$ & $114(14.0 \%)$ \\
\hline Been upset & $0.46 \pm 0.938$ & $0-4$ & $11(1.4 \%)$ & $141(17.3 \%)$ \\
Felt guilty & $0.16 \pm 0.564$ & $0-4$ & $8(1.0 \%)$ & $47(5.8 \%)$ \\
Taken time off work & $0.14 \pm 0.560$ & $0-4$ & $9(1.1 \%)$ & $41(5.0 \%)$ \\
Financial impact & & &
\end{tabular}

No significant associations were found between the presence of TDI and isolated items on the ECOHIS (Table 3).

Table 3. Frequency distribution of preschool children with or without TDI according to each ECOHIS item.

\begin{tabular}{lccccccc}
\hline \multicolumn{1}{c}{ ECOHIS } & \multicolumn{2}{c}{ Yes } & \multicolumn{2}{c}{ No } & \multicolumn{2}{c}{ Total } & $p$-value \\
\hline Domains, Items & $\mathrm{N}$ & $\%$ & $\mathrm{~N}$ & $\%$ & $\mathrm{~N}$ & $\%$ & \\
\hline - Child Impact & & & & & & & \\
\hline No impact & 185 & $(33.0)$ & 376 & $(67.0)$ & 561 & $(68.9)$ & 0.45 \\
$\quad$ Impact & 92 & $(36.4)$ & 161 & $(63.6)$ & 253 & $(31.1)$ & \\
\hline - Symptoms Domain & & & & & & & \\
\hline Related to Pain & & & & & & & \\
No impact & 218 & $(34.2)$ & 420 & $(65.8)$ & 638 & $(78.4)$ & 0.873 \\
Impact & 59 & $(33.5)$ & 117 & $(66.5)$ & 176 & $(21.6)$ & \\
\hline
\end{tabular}


Table 3. Cont.

\begin{tabular}{|c|c|c|c|c|c|c|c|}
\hline \multirow[t]{2}{*}{ ECOHIS } & \multicolumn{7}{|c|}{ TDI } \\
\hline & \multicolumn{2}{|c|}{ Yes } & \multicolumn{2}{|c|}{ No } & \multicolumn{2}{|c|}{ Total } & $p$-value \\
\hline \multicolumn{8}{|l|}{ - Function Domain } \\
\hline \multicolumn{8}{|c|}{ Had difficulty drinking hot or cold beverages } \\
\hline No impact & 236 & $(33.6)$ & 467 & $(66.4)$ & 703 & $(87.4)$ & 0.543 \\
\hline Impact & 37 & $(36.6)$ & 64 & $(63.4)$ & 101 & $(12.6)$ & \\
\hline \multicolumn{8}{|c|}{ Had difficulty eating some food } \\
\hline No impact & 235 & $(33.5)$ & 466 & $(66.5)$ & 462 & $(81.9)$ & 0.724 \\
\hline Impact & 36 & $(35.3)$ & 66 & $(64.7)$ & 102 & $(18.1)$ & \\
\hline \multicolumn{8}{|c|}{ Had difficulty pronouncing words } \\
\hline No impact & 239 & $(32.7)$ & 491 & $(67.3)$ & 730 & $(92.5)$ & 0.213 \\
\hline Impact & 24 & $(40.7)$ & 35 & $(59.3)$ & 59 & $(7.5)$ & \\
\hline \multicolumn{8}{|l|}{ Missing preschool } \\
\hline No impact & 266 & $(33.9)$ & 519 & $(66.1)$ & 785 & $(96.4)$ & 0.652 \\
\hline Impact & 11 & $(37.9)$ & 18 & $(62.1)$ & 29 & $(3.6)$ & \\
\hline \multicolumn{8}{|l|}{ - Psychological Domain } \\
\hline \multicolumn{8}{|l|}{ Had trouble sleeping } \\
\hline No impact & 257 & $(33.8)$ & 504 & $(66.2)$ & 761 & $(94.4)$ & 0.952 \\
\hline Impact & 15 & $(33.3)$ & 30 & $(66.7)$ & 45 & $(5.6)$ & \\
\hline \multicolumn{8}{|c|}{ Been irritable or frustrated } \\
\hline No impact & 244 & $(33.8)$ & 477 & $(66.2)$ & 721 & $(95.7)$ & 0.959 \\
\hline Impact & 29 & $(34.1)$ & 56 & $(65.9)$ & 32 & $(4.3)$ & \\
\hline \multicolumn{8}{|c|}{ - Self-image/Social Interaction Domain } \\
\hline \multicolumn{8}{|c|}{ Avoided smiling or laughing } \\
\hline No impact & 264 & $(63.6)$ & 522 & $(66.4)$ & 786 & $(97.8)$ & 0.336 \\
\hline Impact & 8 & $(44.8)$ & 10 & $(55.6)$ & 18 & $(2.2)$ & \\
\hline \multicolumn{8}{|l|}{ Avoided talked } \\
\hline No impact & 265 & $(33.7)$ & 521 & $(66.3)$ & 786 & $(97.7)$ & 0.445 \\
\hline Impact & 8 & $(42.1)$ & 11 & $(57.9)$ & 19 & $(2.3)$ & \\
\hline \multicolumn{8}{|l|}{ Family Impact } \\
\hline No impact & 205 & $(33.7)$ & 403 & $(66.3)$ & 608 & $(75.1)$ & 0.874 \\
\hline Impact & 69 & $(34.3)$ & 132 & $(65.7)$ & 201 & $(24.9)$ & \\
\hline \multicolumn{8}{|l|}{ - Distress Domain } \\
\hline \multicolumn{8}{|l|}{ Been upset } \\
\hline No impact & 229 & $(33.2)$ & 460 & $(66.8)$ & 689 & $(85.8)$ & 0.349 \\
\hline Impact & 43 & $(37.7)$ & 71 & $(62.3)$ & 114 & $(14.2)$ & \\
\hline \multicolumn{8}{|l|}{ Felt guilty } \\
\hline No impact & 227 & $(34.3)$ & 435 & $(65.7)$ & 662 & $(82.4)$ & 0.705 \\
\hline Impact & 46 & $(32.6)$ & 95 & $(67.4)$ & 141 & $(17.6)$ & \\
\hline \multicolumn{8}{|c|}{ - Family Function Domain } \\
\hline \multicolumn{8}{|l|}{ Taken time off work } \\
\hline No impact & 256 & $(33.7)$ & 503 & $(66.3)$ & 759 & $(94.2)$ & 0.521 \\
\hline Impact & 18 & $(38.3)$ & 29 & $(61.7)$ & 47 & $(5.8)$ & \\
\hline Financial Impact & & & & & & & \\
\hline No impact & 260 & $(34.0)$ & 504 & $(66.0)$ & 764 & $(94.9)$ & 0.759 \\
\hline Impact & 13 & $(31.7)$ & 28 & $(68.3)$ & 41 & $(5.1)$ & \\
\hline
\end{tabular}


In the bivariate analysis, the following variables were associated with the prevalence of impact on the quality of life of the child: lower level of mother's schooling; lower household income; greater number of residents in the home; attending a private preschool/daycare center; poorer evaluation of parents/caregivers regarding child's general and oral health; presence of TDI; tooth discoloration; luxation; history of visiting the dentist; and history of toothache. However, only the parent/caregiver's evaluation regarding the child's oral health and a history of toothache remained in the final Poisson multiple regression model (Table 4).

Table 4. Frequency distribution and Poisson regression analysis according to independent variables and impact on quality of life (QoL) of preschool children.

\begin{tabular}{|c|c|c|c|c|c|c|}
\hline \multirow[t]{3}{*}{ Variable } & \multicolumn{2}{|c|}{ Impact on child's QoL } & \multicolumn{2}{|c|}{ Bivariate } & \multirow{2}{*}{\multicolumn{2}{|c|}{$\begin{array}{c}\text { Multivariate } \\
\text { Adjusted prevalence ratio }\end{array}$}} \\
\hline & \multirow{2}{*}{$\begin{array}{c}\text { Present } \\
\text { n (\%) }\end{array}$} & \multirow{2}{*}{$\begin{array}{c}\text { Absent } \\
\text { n (\%) }\end{array}$} & \multicolumn{2}{|c|}{ Unadjusted prevalence ratio } & & \\
\hline & & & $p$-value & $(95 \% \mathrm{CI})$ & $p$-value & $(95 \% \mathrm{CI})$ \\
\hline \multicolumn{7}{|l|}{ - Gender of child } \\
\hline Male & $131(31.0)$ & $291(69.0)$ & & 1.00 & - & - \\
\hline Female & $122(31.1)$ & $270(68.9)$ & 0.980 & $\begin{array}{c}1.003 \\
(0.817-1.230) \\
\end{array}$ & - & - \\
\hline \multicolumn{7}{|c|}{ - Mother's schooling } \\
\hline$>8$ years of study & $113(25.9)$ & $324(74.1)$ & & 1.00 & - & - \\
\hline$\leq 8$ years of study & $139(37.3)$ & $234(62.7)$ & 0.001 & $\begin{array}{c}1.441 \\
(1.173-1.771) \\
\end{array}$ & - & - \\
\hline \multicolumn{7}{|c|}{ - Monthly household income } \\
\hline $\begin{array}{l}>3 \text { times the } \\
\text { minimum wage }\end{array}$ & $29(20.1)$ & $115(79.9)$ & & 1.00 & - & - \\
\hline $\begin{array}{l}\leq 3 \text { times the } \\
\text { minimum wage }\end{array}$ & $219(34.5)$ & $415(65.5)$ & 0.020 & $\begin{array}{c}1.715 \\
(1.218-2.416) \\
\end{array}$ & - & - \\
\hline \multicolumn{7}{|c|}{ - $\mathrm{N}^{\circ}$ of residents in home } \\
\hline$<6$ & $199(29.5)$ & $475(70.5)$ & & 1.00 & - & - \\
\hline$\geq 6$ & $50(40.7)$ & $73(59.3)$ & 0.010 & $\begin{array}{c}1.377 \\
(1.079-1.756) \\
\end{array}$ & - & - \\
\hline \multicolumn{7}{|l|}{ - Type of school } \\
\hline Public & $153(34.9)$ & $285(65.1)$ & & 1.00 & - & - \\
\hline Private & $100(26.6)$ & $276(73.4)$ & 0.011 & $\begin{array}{c}1.313 \\
(1.064-1.622) \\
\end{array}$ & - & - \\
\hline \multicolumn{7}{|c|}{ - Caregiver's perception of child's general health } \\
\hline Good & $247(30.8)$ & $554(69.2)$ & & 1.00 & & - \\
\hline Poor & $6(75.0)$ & $2(25.0)$ & 0.001 & $\begin{array}{c}2.432 \\
(1.609-3.677) \\
\end{array}$ & - & - \\
\hline \multicolumn{7}{|c|}{ - Caregiver's perception of child's oral health } \\
\hline Good & $205(27.0)$ & $554(73.0)$ & & 1.00 & & 1.00 \\
\hline Poor & $48(88.9)$ & $6(11.1)$ & 0.001 & $\begin{array}{c}2.432 \\
(1.609-3.677) \\
\end{array}$ & 0.23 & $\begin{array}{c}1.210 \\
(1.027-1.426) \\
\end{array}$ \\
\hline
\end{tabular}


Table 4. Cont.

\begin{tabular}{|c|c|c|c|c|c|c|}
\hline \multirow[t]{3}{*}{ Variable } & \multicolumn{2}{|c|}{ Impact on child's QoL } & \multicolumn{2}{|c|}{ Bivariate } & \multicolumn{2}{|c|}{ Multivariate } \\
\hline & \multirow{2}{*}{$\begin{array}{c}\text { Present } \\
\text { n (\%) }\end{array}$} & \multirow{2}{*}{$\begin{array}{c}\text { Absent } \\
\text { n (\%) }\end{array}$} & \multicolumn{2}{|c|}{ Unadjusted prevalence ratio } & \multicolumn{2}{|c|}{ Adjusted prevalence ratio } \\
\hline & & & $p$-value & $(95 \% \mathrm{CI})$ & $p$-value & $(95 \% \mathrm{CI})$ \\
\hline \multicolumn{7}{|l|}{ - TDI } \\
\hline Yes & $95(33.8)$ & $186(66.2)$ & & 1.00 & - & - \\
\hline No & $158(29.6)$ & $357(70.4)$ & 0.342 & $\begin{array}{c}1.108 \\
(0.897-1.368)\end{array}$ & - & - \\
\hline \multicolumn{7}{|l|}{ - Type of trauma } \\
\hline $\begin{array}{l}\text { Avulsion/ } \\
\text { Luxation }\end{array}$ & $6(46.2)$ & $7(53.8)$ & 0.139 & $\begin{array}{c}1.572 \\
(0.864-2.861)\end{array}$ & - & - \\
\hline Discoloration & $37(40.7)$ & $54(59.3)$ & 0.020 & $\begin{array}{c}1385 \\
(1.052-1.822)\end{array}$ & - & - \\
\hline $\begin{array}{l}\text { Enamel + dentin } \\
\text { fracture }\end{array}$ & $13(33.3)$ & $26(66.7)$ & 0.588 & $\begin{array}{c}1.135 \\
(0.717-1.797)\end{array}$ & - & - \\
\hline $\begin{array}{l}\text { Enamel fracture } \\
\text { or no trauma }\end{array}$ & $197(29.4)$ & $474(70.6)$ & & 1.00 & - & - \\
\hline \multicolumn{7}{|c|}{ - Number of teeth with trauma } \\
\hline None & $158(29.6)$ & $375(70.4)$ & & 1.00 & - & - \\
\hline One & $52(29.4)$ & $125(70.6)$ & 0.947 & $\begin{array}{c}0.991 \\
(0.762-1.289)\end{array}$ & - & - \\
\hline Two or more & $43(41.3)$ & $61(58.7)$ & 0.013 & $\begin{array}{c}1.395 \\
(1.072-1.816) \\
\end{array}$ & - & - \\
\hline \multicolumn{7}{|l|}{ - Dental caries } \\
\hline No & $45(16.1)$ & $235(83.9)$ & $<0.001$ & $\begin{array}{c}2.424 \\
(1.817-3.232)\end{array}$ & - & - \\
\hline Yes & $208(39.0)$ & $326(61.0)$ & & 1.00 & - & - \\
\hline \multicolumn{7}{|l|}{ - Malocclusion } \\
\hline No & $80(28.6)$ & $200(71.4)$ & & 1.00 & - & - \\
\hline Yes & $173(32.4)$ & $361(67.6)$ & 0.267 & $\begin{array}{c}1.134 \\
(0.908-1.416) \\
\end{array}$ & - & - \\
\hline \multicolumn{7}{|c|}{ - History of toothache } \\
\hline No & $15(12.2)$ & $108(87.8)$ & - & 1.00 & - & 1.00 \\
\hline Yes & $62(87.3)$ & $9(12.7)$ & 0.001 & $\begin{array}{c}7.161 \\
(4.420-11.600) \\
\end{array}$ & 0.001 & $\begin{array}{c}4.997 \\
(2.943-8.483)\end{array}$ \\
\hline \multicolumn{7}{|c|}{ - History of visits to dentist } \\
\hline No & $173(28.2)$ & $441(71.8)$ & & 1.00 & - & - \\
\hline Yes & $78(39.8)$ & $118(60.2)$ & 0.002 & $\begin{array}{c}1.412 \\
(1.41-1.749)\end{array}$ & - & - \\
\hline
\end{tabular}

In the bivariate analysis, the following variables were associated the prevalence of impact on the quality of life of the family: lower level of the mother's schooling; greater number of residents in the home; poorer evaluation of parents/caregivers regarding child's general and oral health; tooth discoloration; luxation; history of visiting the dentist; and history of toothache. However, only history of toothache remained in the final Poisson multiple regression model (Table 5). 
Table 5. Frequency distribution and Poisson regression analysis according to independent variables and impact on quality of life (QoL) of family of preschoolers.

\begin{tabular}{|c|c|c|c|c|c|c|}
\hline \multirow[t]{3}{*}{ Variable } & \multicolumn{2}{|c|}{ Impact on family's QoL } & \multicolumn{2}{|c|}{ Bivariate } & \multirow{2}{*}{\multicolumn{2}{|c|}{$\begin{array}{c}\text { Multivariate } \\
\text { Adjusted prevalence ratio }\end{array}$}} \\
\hline & \multirow{2}{*}{$\begin{array}{c}\text { Present } \\
\text { n }(\%)\end{array}$} & \multirow{2}{*}{$\frac{\text { Absent }}{\text { n (\%) }}$} & \multicolumn{2}{|c|}{ Unadjusted prevalence ratio } & & \\
\hline & & & $p$-value & $(95 \% \mathrm{CI})$ & $p$-value & $(95 \% \mathrm{CI})$ \\
\hline \multicolumn{7}{|l|}{ - Gender of child } \\
\hline Male & $94(24.2)$ & $294(75.8)$ & & 1.00 & - & - \\
\hline Female & $107(25.4)$ & $314(74.6)$ & 0.696 & $\begin{array}{c}1.049 \\
(0.825-1.334)\end{array}$ & - & - \\
\hline \multicolumn{7}{|c|}{ - Mother's schooling } \\
\hline $\begin{array}{l}>8 \text { years } \\
\text { of study }\end{array}$ & $97(22.3)$ & $338(77.7)$ & & 1.00 & - & - \\
\hline $\begin{array}{l}\leq 8 \text { years } \\
\text { of study }\end{array}$ & $103(27.8)$ & $267(72,2)$ & 0,070 & $\begin{array}{c}1.248 \\
(0.982-1.587) \\
\end{array}$ & - & - \\
\hline \multicolumn{7}{|c|}{ - Monthly household income } \\
\hline $\begin{array}{l}>3 \text { times the } \\
\text { minimum wage }\end{array}$ & $31(21.5)$ & $113(78.5)$ & & 1.00 & - & - \\
\hline $\begin{array}{l}\leq 3 \text { times the } \\
\text { minimum wage }\end{array}$ & $165(26.2)$ & $464(73.8)$ & 0.252 & $\begin{array}{c}1.219 \\
(0.869-1.709) \\
\end{array}$ & - & - \\
\hline \multicolumn{7}{|c|}{ - $N^{\circ}$ of residents in home } \\
\hline$<6$ & $160(23.9)$ & $510(76.1)$ & & 1.00 & - & - \\
\hline$\geq 6$ & $38(30.9)$ & $85(69.1)$ & 0.089 & $\begin{array}{c}1.294 \\
(0.961-1.741) \\
\end{array}$ & - & - \\
\hline \multicolumn{7}{|l|}{ - Type of school } \\
\hline Public & $85(22.8)$ & $287(77.2)$ & & 1.00 & - & - \\
\hline Private & $116(26.5)$ & $321(73.5)$ & 0.227 & $\begin{array}{c}1.162 \\
(0.911-1.482) \\
\end{array}$ & - & - \\
\hline \multicolumn{7}{|c|}{ - Caregiver's perception of child's general health } \\
\hline Good & $194(24.4)$ & $602(75.6)$ & & 1.00 & - & - \\
\hline Poor & $5(62.5)$ & $3(37.5)$ & 0.001 & $\begin{array}{c}2.564 \\
(1.479-4.447) \\
\end{array}$ & - & - \\
\hline \multicolumn{7}{|c|}{ - Caregiver's perception of child's oral health } \\
\hline Good & $161(21.4)$ & $593(78.6)$ & & 1.00 & - & - \\
\hline Poor & $40(74.1)$ & $14(25.9)$ & 0.001 & $\begin{array}{c}3.469 \\
(2.815-4.275) \\
\end{array}$ & - & - \\
\hline \multicolumn{7}{|l|}{ - TDI } \\
\hline Yes & $131(24.7)$ & $400(75.3)$ & & 1.00 & - & - \\
\hline No & $70(25.2)$ & $208(74.8)$ & 0.874 & $\begin{array}{c}1.121 \\
(0.793-1.313)\end{array}$ & & - \\
\hline
\end{tabular}


Table 5. Cont.

\begin{tabular}{|c|c|c|c|c|c|c|}
\hline \multirow[t]{3}{*}{ Variable } & \multicolumn{2}{|c|}{ Impact on family's QoL } & \multicolumn{2}{|c|}{ Bivariate } & \multicolumn{2}{|c|}{ Multivariate } \\
\hline & \multirow{2}{*}{$\begin{array}{c}\text { Present } \\
\text { n (\%) }\end{array}$} & \multirow{2}{*}{$\begin{array}{c}\text { Absent } \\
\text { n (\%) }\end{array}$} & \multicolumn{2}{|c|}{ Unadjusted prevalence ratio } & \multicolumn{2}{|c|}{ Adjusted prevalence ratio } \\
\hline & & & $p$-value & $(95 \% \mathrm{CI})$ & $p$-value & $(95 \% \mathrm{CI})$ \\
\hline \multicolumn{7}{|l|}{ - Type of trauma } \\
\hline $\begin{array}{l}\text { Avulsion/ } \\
\text { Luxation }\end{array}$ & $5(38.5)$ & $8(61.5)$ & 0.185 & $\begin{array}{c}1.606 \\
(0.797-3.236)\end{array}$ & - & - \\
\hline Discoloration & $28(31.5)$ & $61(68.5)$ & 0.111 & $\begin{array}{c}1.313 \\
(0.939-1.836)\end{array}$ & - & - \\
\hline $\begin{array}{l}\text { Enamel }+ \\
\text { dentin fracture }\end{array}$ & $8(20.5)$ & $31(79.5)$ & 0.631 & $\begin{array}{c}0.856 \\
(0.455-1.612)\end{array}$ & - & - \\
\hline $\begin{array}{l}\text { Enamel } \\
\text { fracture or } \\
\text { no trauma }\end{array}$ & $160(24.0)$ & $508(76.0)$ & & 1.00 & - & - \\
\hline \multicolumn{7}{|c|}{ - Number of teeth with trauma } \\
\hline None & $131(24.7)$ & $400(75.3)$ & & 1.00 & - & - \\
\hline One & $40(23.0)$ & $134(77.0)$ & 0.655 & $\begin{array}{c}0.932 \\
(0.683-1.270)\end{array}$ & - & - \\
\hline Two or more & $30(28.8)$ & $74(71.2)$ & 0.362 & $\begin{array}{c}1.169 \\
(0.835-1.637) \\
\end{array}$ & - & - \\
\hline \multicolumn{7}{|l|}{ - Dental caries } \\
\hline No & $34(12.3)$ & $243(87.7)$ & $<0.001$ & $\begin{array}{c}2.557 \\
(1.822-3.589)\end{array}$ & 0.022 & $\begin{array}{c}2.305 \\
(1.130-4.702)\end{array}$ \\
\hline Yes & $167(31.4)$ & $365(68.6)$ & & 1.00 & - & 1.00 \\
\hline \multicolumn{7}{|l|}{ - Malocclusion } \\
\hline No & $61(22.0)$ & $216(78.0)$ & & 1.00 & - & - \\
\hline Yes & $140(26.3)$ & $392(73.7)$ & 0.185 & $\begin{array}{c}1.195 \\
(0.918-1.555) \\
\end{array}$ & - & - \\
\hline \multicolumn{7}{|c|}{ - History of toothache } \\
\hline No & $22(18.0)$ & $100(82.0)$ & - & 1.00 & & 1.00 \\
\hline Yes & $48(67.6)$ & $23(32.4)$ & 0.001 & $\begin{array}{c}3.749 \\
(2.485-5.656)\end{array}$ & 0.001 & $\begin{array}{c}2.791 \\
(1.801-4.325)\end{array}$ \\
\hline \multicolumn{7}{|c|}{ - History of visits to dentist } \\
\hline No & $129(21.1)$ & $481(78.9)$ & & 1.00 & - & - \\
\hline Yes & $71(36.4)$ & $124(63.6)$ & 0.001 & $\begin{array}{c}1.772 \\
(1.354-2.190)\end{array}$ & - & - \\
\hline
\end{tabular}

\section{Discussion}

OHRQoL assessment tools have been employed with increasing frequency in oral health surveys [32]. Dental disease and treatment experience can negatively affect the OHRQL of preschool children and their parents/caregivers [6]. The ECOHIS is a proxy measure of children's OHRQoL [8] for which parents/caregivers are the secondary respondents, as it is believed that very young children do not have sufficient cognitive skills to evaluate their own quality of life [6,24]. This method has been validated in the existing literature $[6,8,22,33]$. 
The prevalence of negative impact on quality of life of the child was $31.1 \%$, which is lower than the figure reported in previous Brazilian studies (49\% to 69.3\%) [5,17,18]. The divergences may be explained by differences in the sample profile and methods employed. In studies with a prevalence rate as high as $69.3 \%$ [5,17], the samples were not population-based and were made up of children treated at healthcare services, which may have influenced the responses. In the study with a prevalence rate of $49 \%$ [18], the sample was randomly selected but not representative. Moreover, in all three studies, responses of "hardly ever" were recorded as the presence of impact, which likely increased the prevalence of negative impact on quality of life. In the present study, the decision was made to consider the presence of impact only when answers of "occasionally", "often", and "very often" were recorded [6]. This point merits attention, as previous investigations have not defined the cutoff point for the presence/absence of a negative impact on quality of life using the ECOHIS. Some studies use the mean or median ECOHIS score to determine the negative impact and associations with the variables analyzed [7,17,34,35]. Other studies use a qualitative categorization (yes/no), but with adaptation to the questionnaire (combination of items) [36] or cutoff points that differ from that used in the present study $[17,18,35]$, which hinders the comparison of the results.

In the analysis, the most frequent responses on the Child Impact Section of the ECOHIS included "related to pain", "had difficulty drinking hot or cold beverages", and "had difficulty eating some foods". This is similar to findings reported in other Brazilian studies $[8,18,35]$ as well as a study carried out in China [7]. The symptom and function domains seem to be more perceptible to family members than the domains concerning psychology and self-image. On the other hand, studies report that children in this age group do not have sufficient psychological maturity or self-image to establish comparisons with other children; thus, the consequences of oral conditions may be minimized with regard to these aspects [5,37]. The structure of a child's self-concept is age dependent as a result of continuous cognitive, emotional, social and language development [38]. Child developmental psychology explains that the age of six years is marked by the onset of abstract thinking and self-awareness [37]. Indeed, emotional status and aesthetics are considered to be factors associated with a negative impact on quality of life among older age groups. A Brazilian study involving adolescents aged 11 to 14 years using the Child Perceptions Questionnaire (CPQ11-14) found that adolescents with aesthetic abnormalities experienced a negative impact on social wellbeing, mainly with regard to avoid smiling or laughing and being concerned about what other people may think or say [3]. A similar finding is reported in a study carried out in Great Britain involving adolescents aged 16 and 17 years using the Oral Health Impact Profile (OHIP-14), in which aesthetic abnormalities constituted a major self-reported cause of impact on daily performances [39]. However, the comparison of quality of life studies involving different age groups is hampered by the methods employed. While studies involving adolescents employ self-reports of impact, investigations involving children aged five years or younger rely on proxy reports from parents/guardians which may exert an influence on the results [18]. Indeed, there are reports in the literature regarding the lack of importance some parents/caregivers place on the primary dentition $[13,40]$, which also may have contributed to the findings.

The prevalence of negative impact on the quality of life of the family was $24.7 \%$. Once again, this is lower than the figure reported in previous Brazilian studies $(30.7 \%$ to $35 \%)[5,17,18]$. The reasons for these divergences are likely the same as those reported with regard to the Child Impact Section. 
Another cross-sectional study carried out in Brazil specifically analyzing the impact of oral health conditions among children on families reports a negative impact of $87.3 \%$ [35]. However, the study cited was conducted at a healthcare service involving children with a high percentage of accumulated treatment needs, which likely influenced the results. In contrast, the present study involved a population-based sample at preschools.

In analyzing each item in the Family Health Section, "felt guilty" and "been upset" were the most cited, which is in line with a tendency observed in previous studies [5,7,18]. In an investigation conducted in the United States, the most frequent responses were "taken time off work" and "felt guilty" [6]. The greater participation of women in the job market in recent decades, which has led to delegating the care of children's health to third parties [41], and the lack of knowledge regarding the need to visit the dentist in the early years of a child's life [42] likely affect the feelings of parents/caregivers in terms of the negative impact on the quality of life of the family. A number of authors report that mothers are aware of their responsibilities with regard to providing oral health care and express feelings of guilt, concern, anger, and despair associated with the adverse oral conditions of their children $[43,44]$.

The prevalence of TDI was $34.6 \%$ and the characterization of trauma was in agreement with that described in the literature $[12,13,45]$. It should be pointed out that this prevalence rate may have been underestimated due to the self-correction of some types of past trauma, which were therefore not diagnosed at the time of the exam. This constitutes one of the limitations of the cross-sectional study design. TDI was not significantly associated with the domains of the ECOHIS. Most cases of trauma were mild fractures, which may have influenced the results. Likewise, previous studies have found no association between TDI and the different domains of the ECOHIS [18,35] and also report mild trauma in most cases. However, one cannot discard the possibility of memory bias [46], which is a limitation of cross-sectional studies; many parents/caregivers may have forgotten the details related to the time at which the traumatic event occurred. Another Brazilian study found an association between severe TDI (complicated crown fracture) in the primary dentition and a negative impact on quality of life [17]. The study cited was carried out at a healthcare service and, according to the authors, the association between TDI and negative impact on quality of life was likely due to symptoms frequently related to complicated TDI, such as pain, irritation, difficulty eating some foods, trouble sleeping, and difficulty drinking hot or cold beverages.

The Poisson multivariate analysis controlled for the presence of dental caries and malocclusion. Thus only a parent/caregiver's evaluation of the child's oral health and a history of toothache remained associated with a negative impact on quality of life in the Child Section of the ECOHIS. For public health purposes, the evaluations of parents/caregivers regarding their children's oral health should be incorporated into population-based surveys aimed at assessing the need for dental care among children of preschool age [9]. Children's self perceptions of their oral own health status was reported to be a strong predictor of negative impact on quality of life in a Brazilian study employing the CPQ11-14 [1]. A study carried out in the United States with children aged four to 12 years of age which used the Michigan Oral Health-Related Quality of Life Scale reports that poor oral health may prevent children from expressing positive emotions, which can impact their social interactions and the way they feel about themselves [47]. A validation study for the Oral Impacts on Daily Performance scale for children (Child-OIDP) carried out in Saudi Arabia involving 12-year-olds found that the perception of oral 
health plays an important role in the determination of negative impact on quality of life [4]. Regarding a history of toothache, previous Brazilian studies point to a strong association between this variable and the search for dental treatment in preschool children $[43,48]$. Toothache was the most frequently associated cause of nearly all impacts in both private and public school attendees in the Saudi Arabian study using the Child-OIDP on 12-year-olds [4]. In India, a study reports an $85 \%$ prevalence rate of negative impact on activities of daily living due to toothache among 12-year-olds [49]. In a study with a similar methodology as that employed in the present investigation [18], the variables associated with a negative impact on quality of life in the Child Impact Section were the presence of caries, social indicators, and a history of trauma reported by parents/caregivers. However, the normative diagnosis of TDI was not associated with a negative impact on quality of life and the authors did not investigate a history of toothache or oral health perceptions. In the present study, the normative diagnosis of trauma was considered rather than the perception of trauma reported by parents/caregivers, which likely influenced the findings and could be considered a limitation of this study, as the impact on quality of life occurs beginning with the moment at which the trauma is perceived by the parent/caregiver and not when diagnosed by the dentist. Nonetheless, other authors have employed this same methodology $[5,17,35]$.

On the Family Impact Section, dental caries and history of toothache were the variables associated with a negative impact on quality of life in the multivariate model, further demonstrating the value of this factor. The disregard for the deciduous dentition as well the lack of knowledge on the need to visit the dentist in the early years of a child's life and the etiological factors of adverse oral conditions may have contributed to this finding $[43,48]$. Indeed, a number of Brazilian studies report low rates of seeking dental care for children aged five years or younger $[24,42,48]$. Thus, greater investment is needed to raise awareness among parents/caregivers regarding the importance of the primary dentition and routine dental care for preschool children. The greater frequency of responses of "felt guilty" and "been upset" may reflect this result and may be related to untreated caries rather than TDI per se, as most cases of dental trauma were mild and not associated with a negative impact on quality of life.

Although social indicators have been reported to be predisposing factors for impact on the quality of life of preschoolers $[5,7,8,35]$, no such association was found in the present study. The studies cited were carried out in different regions/countries, which may have influenced the results. Predictors of a negative impact on quality of life may vary across populations and these aspects should be taken into account in decision-making processes regarding the allocation of resources for healthcare programs [50].

\section{Conclusions}

The present findings reveal that TDI exerted no negative impact on the quality of life of preschoolers and their families. As this investigation was a population-based study, the findings may be extrapolated to the population. The evaluation of parents/caregivers regarding the oral health of their children and a history of toothache were associated with a negative impact on quality of life of the Children Impact Section. Dental caries and history of toothache were associated with a negative impact on the Family Impact Section. 


\section{Acknowledgments}

This study was supported by the State University of Paraíba, the Brazilian Coordination of Higher Education, Ministry of Education (CAPES), the Research Foundation of the State of Minas Gerais (FAPEMIG) and the National Council for Scientific and Technological Development (CNPQ), Brazil.

\section{Conflicts of Interest}

The authors declare no conflict of interest.

\section{References}

1. Paula, J.S.; Leite, I.C.; Almeida, A.B.; Ambrosano, G.M.; Pereira, A.C.; Mialhe, F.L. The influence of oral health conditions, socioeconomic status and home environment factors on schoolchildren's self-perception of quality of life. Health Qual. Life Outcomes 2012, 10, 6-13.

2. Barbosa, T.S.; Gavião, M.B. Oral health-related quality of life in children: Part II. Effects of clinical oral health status. A systematic review. Int. J. Dent. Hyg. 2008, 6, 100-107.

3. Bendo, C.B.; Paiva, S.M.; Torres, C.T.; Oliveira, A.C.; Goursand, D.; Pordeus, I.A. Association between treated/untreated traumatic dental injuries and impact on quality of life of Brazilian schoolchildren. Health Qual. Life Outcomes 2010, 114, 1-8.

4. Nurelhuda, N.M.; Ahmed, M.F.; Trovik, T.A.; Astrom, A.N. Evaluation of oral health-related quality of life among Sudanese schoolchildren using Child-OIDO inventory. Health Qual. Life Outcomes 2010, 8, 152, doi:10.1186/1477-7525-8-152.

5. Abanto, J.; Carvalho, T.S.; Mendes, F.M.; Wanderley, M.T.; Bönecker, M.; Raggio, D.P. Impact of oral diseases and disorders on oral health-related quality of life of preschool children. Community Dent. Oral Epidemiol. 2011, 39, 105-114.

6. Pahel, B.T.; Rozier, R.G.; Salde, G.D. Parental perceptions of children's oral health: The Early Childhood Oral Health Impact Scale (ECohis). Health Qual. Life Outcomes 2007, 5, 6, doi:10.1186/1477-7525-5-6.

7. Wong, H.M.; McGrath, C.P.; King, N.M. Rasch validation of the early childhood oral health impact scale. Community Dent. Oral Epidemiol. 2011, 39, 449-457.

8. Scarpelli, A.C.; Oliveira, B.H.; Tesch, F.C.; Leão, A.T.; Pordeus, I.A.; Paiva, S.M. Psychometric properties of the Brazilian version of the Early Childhood Oral Health Impact Scale (B-ECOHIS). BMC Oral Health 2011, 11, 19, doi:10.1186/1472-6831-11-19.

9. Talekar, B.S.; Rozier, R.G.; Slade, G.D.; Ennett, S.T. Parental perceptions of their preschool-aged children's oral health. J. Am. Dent. Assoc. 2005, 136, 364-372.

10. Sgan-Cohen, H.D.; Megnagi, G.; Jacobi, Y. Dental trauma and its association with anatomic, behavioral, and social variables among fifth and sixth grade schoolchildren in Jerusalem. Community Dent. Oral Epidemiol. 2005, 33, 174-180.

11. Kramer, P.F.; Zembruski, C.; Ferreira, S.H.; Feldens, C.A. Traumatic dental injuries in Brazilian preschool children. Dent. Traumatol. 2003, 19, 299-303.

12. Granville-Garcia, A.F.; de Menezes, V.A.; de Lira, P.I. Dental trauma and associated factors in Brazilian preschoolers. Dent. Traumatol. 2006, 22, 318-322. 
13. Robson, F.; Ramos-Jorge, M.L.; Bendo, C.B.; Vale, M.P.; Paiva, S.M.; Pordeus, I.A. Prevalence and determining factors of traumatic injuries to primary teeth in preschool children. Dent. Traumatol. 2009, 25, 118-122.

14. Granville-Garcia, A.F.; Vieira, I.T.; Siqueira, M.J.; de Menezes, V.A.; Cavalcanti, A.L. Traumatic dental injuries and associated factors among Brazilian preschool children aged 1-5 years. Acta Odontol. Latinoam. 2010, 23, 47-52.

15. Viegas, C.M.; Scarpelli, A.C.; Carvalho, A.C.; Ferreira, F.M.; Pordeus, I.A.; Paiva, S.M. Predisposing factors for traumatic dental injuries in Brazilian preschool children. Eur. Arch. Paediatr. Dent. 2010, 11, 59-65.

16. Ferreira, J.M.; Andrade, E.M.F.; Katz, C.R.; Rosenblatt, A. Prevalence of dental trauma in deciduous teeth of Brazilian children. Dent. Traumatol. 2009, 25, 219-223.

17. Aldrigui, J.M.; Abanto, J.; Carvalho, T.S.; Mendes, F.M.; Wanderley, M.T.; Bönecker, M.; Raggio, D.P. Impact of traumatic dental injuries and malocclusions on quality of life of young children. Health Qual. Life Outcomes 2011, 24, 78, doi:10.1186/1477-7525-9-78.

18. Viegas, C.M.; Scarpelli, A.C.; Carvalho, A.C.; Ferreira, F.M.; Pordeus, I.A.; Paiva, S.M. Impact of traumatic dental injury on quality of life among Brazilian preschool children and their families. Pediatr. Dent. 2012, 36, 300-307.

19. Brazilian Institute of Geography and Statistics (IBGE). First Results of the 2010 Census. Avaulable online: http://www.censo2010.ibge.gov.br/dadosdivulgados/index.php?uf=25 (accessed on 3 August 2012).

20. David, J.; Astrom, A.; Wang, N.J. Factors associated with traumatic dental injuries among 12-year-old schoolchildren in South India. Dent. Traumatol. 2009, 25, 500-505.

21. Altman, D.G. Practical Statistics for Medical Research, 1st ed.; Chapman and Hall: London, UK, 1991.

22. Tesch, F.C.; Oliveira, B.H.; Leão, A. Semantic equivalence of the Brazilian version of the Early Chilhood Oral Health Impact Scale. Cad. Saúde Pública 2008, 24, 1897-1909.

23. Tesch, F.C.; Oliveira, B.H.; Leão, A. Measuring the impact of oral health problems on children's quality of life: Conceptual and methodological issues. Cad. Saúde Pública 2007, 23, 2555-2564.

24. Ardenghi, T.M.; Ferreira, F.V.; Piovesan, C.; Mendes, F.M. Age of first dental visit and predictors for oral healthcare utilisation in preschool children. Oral Health Prev. Dent. 2012, 10, 17-27.

25. Burstone, C.J. Lip posture and its significance in treatment planning. Am. J. Orthod. 1967, 53, 262-284.

26. Grabowski, R.; Stahl, F.; Gaebel, M.; Kundt, G. Relationship between occlusal findings and orofacial myofunctional status in primary and mixed dentition. Part I: Prevalence of malocclusions. J. Orofac. Orthop. 2007, 68, 26-37.

27. World Health Organization. Dentition Status and Criteria for Diagnosis and Coding. WHO Oral Health Surveys Basic Methods, 4th ed.; WHO: Geneva, Switzerland, 1997; pp. 39-44

28. Andreasen, J.O.; Andreasen, F.M.; Andersson, L. Textbook and Color Atlas of Traumatic Injuries to the Teeth, 1st ed.; Munskgaard International Publishers: Copenhagen, Denmark, 2007.

29. Ismail, A.I.; Sohn, W.; Tellez, M.; Amaya, A.; Sen, A.; Hasson, H. The International Caries Detection and Assessment System (ICDAS): An integrated system for measuring dental caries. Community Dent. Oral Epidemiol. 2007, 35, 170-178. 
30. Oliveira, A.C.; Paiva, S.M.; Campos, M.R.; Czeresnia, D. Factors associated with malocclusions in children and adolescents with Down syndrome. Am. J. Orthod. Dent. Facial Orthoped. 2008, 133, 489, doi:10.1016/j.ajodo.2007.09.014.

31. Foster, T.D.; Hamilton, M.C. Occlusion in the primary dentition. Study of children at 2 and one-half to 3 years of age. Br. Dent. J. 1969, 126, 76-79.

32. Goursand, D.; Paiva, S.M.; Zarzar, P.M.; Ramos-Jorge, M.L.; Cornacchia, G.M.; Pordeus, I.A.; Allison, P.J. Cross-cultural adaptation of the Child Perceptions Questionnaire 11-14 (CPQ11-14) for the Brazilian Portuguese language. Health Qual. Life Outcomes 2008, 14, 6-12.

33. Locker, D.; Jokovic, A.; Stephens, M.; Kenny, D.; Tompson, B.; Cuyatt, G. Family impact of child oral and oro-facial conditions. Community Dent. Oral Epidemiol. 2002, 30, 438-448.

34. Goettems, M.L.; Ardenghi, T.M.; Romano, A.R.; Demarco, F.F.; Torriani, D.D. Influence of maternal dental anxiety on oral health-related quality of life of preschool children. Qual. Life Res. 2011, 20, 951-959.

35. Abanto, J.; Paiva, S.M.; Raggio, D.P.; Celiberti, P.; Aldrigui, J.M.; Bönecker, M. The impact of dental caries and trauma in children on family quality of life. Community Dent. Oral Epidemiol. 2012, 40, 323-331.

36. Wandera, M.; Kayondo, J.; Engebretsen, I.M.; Okullo, I.; Astrom, A.N. Factors associated with caregivers' perception of children's health and oral health status: A study of 6- to 36-month-olds in Uganda. Int. J. Paediatr. Dent. 2009, 19, 251-262.

37. Hetherington, E.M.; Parke, R.D.; Locke, V.O. Child Psychology: A Contemporary Viewpoint, 1st ed; The McGraw-Hill Companies: New York, NY, USA, 1999.

38. Jokovic, A.; Locker, D.; Stephens, M.; Kenny, D.; Tompson, B.; Guyat, G. Validity and reliability of a questionnaire for measuring child oral-health-related quality of life. J. Dent. Res. 2002, 81, 459-463.

39. Bernabé, E.; Oliveira, C.M.; Sheiham, A. Comparison of the discriminative ability of a generic and a condition-specific OHRQoL measure in adolescents with and without normative need for orthodontic treatment. Health Qual. Life Outcomes 2008, 6, 64, doi:10.1186/1477-7525-6-64.

40. Traebert, J.; Almeida, I.C.; Garghetti, C.; Marcenes, W. Prevalence, need for treatment and predisposing factors of trauma in the permanent dentition of schoolchildren 11-13 years of age. Cad. Saúde Pública 2004, 20, 403-410.

41. Kosmala-Anderson, J.; Wallace, L.M. Breastfeeding works: The role of employers in supporting women who wish to breastfeed and work in four organizations in England. J. Public Health Med. 2006, 28, 183-191.

42. Camargo, M.B.; Barros, A.J.; Frazão, P.; Matijasevich, A.; Santos, I.S.; Peres, M.A.; Peres, K.G. Predictors of dental visits for routine check-ups and for the resolution of problems among preschool children. Rev. Saúde Pública 2012, 46, 87-97.

43. Escobar-Paucar, G.; Sosa-Palacio, C.; Burgos-Gil, L.M. Social representations of pregnant mothers in an urban community: Medellín, Colombia. Salud Pública Méx. 2010, 52, 46-51.

44. Arora, A.; Bedros, D.; Bhole, S.; Do, L.G.; Scott, J.; Blinkhorn, A.; Schwarz, E. Child and family health nurses' experiences of oral health of preschool children: A qualitative approach. J. BMC Public Health 2012, 72, 149-155. 
45. Feldens, C.A.; Kramer, P.F.; Ferreira, S.H.; Spiguel, M.H.; Marquezan, M. Exploring factors associated with traumatic dental injuries in preschool children: A Poisson regression analysis. Dent. Traumatol. 2010, 26, 143-148.

46. Choi, S.C.; Park, J.H.; Pae, A.; Kim, J.R. Retrospective study on traumatic dental injuries in preschool children at Kyung Hee Dental Hospital, Seoul, South Korea. Dent. Traumatol. 2010, 26, 70-75.

47. Patel, R.R.; Tootla, R.; Inglehart, M.R. Does oral health self-perceptions parenteral ratings and video-based assessments of children's smiles? Community Dent. Oral Epidemiol. 2007, 35, 44-52.

48. Goettems, M.L.; Azevedo, M.S.; Correa, M.B.; Costa, C.T.; Wendt, F.P.; Schuch, H.S.; Bonow, M.L.; Romano, A.R.; Torriani, D.D. Dental trauma occurrence and occlusal characteristics in Brazilian preschool children. Pediatr. Dent. 2012, 34, 104-107.

49. Dandi, K.K.; Raom, E.V.; Margabandhu, S. Dental pain as a determinant of expressed need for dental care among 12-year-old school children in India. Indian J. Dent. Res. 2010, 22, 611, doi:10.4103/0970-9290.90320.

50. Buunk-Werkhoven, A.B.; Dijkstra, A. Promoting oral hygiene behavior in recruits in the Dutch army. Mil. Med. 2009, 174, 971-976.

(C) 2013 by the authors; licensee MDPI, Basel, Switzerland. This article is an open access article distributed under the terms and conditions of the Creative Commons Attribution license (http://creativecommons.org/licenses/by/3.0/). 